\title{
A multinational SDI-based system to facilitate disaster risk management in the Andean Community
}

\author{
Martin Molina ${ }^{\mathrm{a}, *}$, Salvador Bayarri ${ }^{\mathrm{b}}$ \\ a Department of Artificial Intelligence, Facultad de Informática, Technical University of Madrid, Campus de Montegancedo S/N, 28660 Boadilla del Monte, Madrid, Spain \\ b IVER Technologies, C/Lérida 20, 46009 Valencia, Spain
}

\section{A R T I C L E I N F O}

\section{Article history:}

Received 27 April 2010

Received in revised form

8 November 2010

Accepted 26 January 2011

Available online 6 April 2011

Keywords:

Spatial Data Infrastructure

Disaster risk management

Geoportal

Thematic search engine

\section{Introduction}

In South America, the Andean region presents conditions that make information management a priority in disaster risk reduction strategies. This is mainly due to the significant frequency of occurrence in this area of potentially disastrous natural phenomena (earthquakes, volcanoes, floods, etc.) together with a growing vulnerability as the urban population expands without appropriate planning. As an answer to this need, the Community of Andean Nations, which includes Bolivia, Colombia, Ecuador, and Peru, developed the Andean Strategy for Disaster Prevention and Relief as a legal framework implemented by the Andean Committee for Disaster Prevention and Relief (CAPRADE). In this context, a project called PREDECAN (Prevention and Mitigation of Disasters in the Andean Community), supported by the European Union and the Community of Andean Nations, was developed with the main goal of building capacities for disaster risk prevention in the Andean Community, in direct relation to the Hyogo Framework for Action 2005-2015 (ISDR, 2005).

One of the goals of this project was the creation of an Andean Information System for Disaster Prevention and Relief (SIAPAD is the acronym of the Spanish name Sistema de Información Andino para la Prevención y Atención de Desastres), an initiative oriented for providing tools for information discovery and visualization,

\footnotetext{
* Corresponding author. Tel.: + 34 913367390; fax: + 34913524819

E-mail addresses: martin.molina@upm.es (M. Molina), salvador.bayarri@iver.es (S. Bayarri).
}

and to facilitate access to the information by various technical organizations of the Andean countries participating in the initiative. We conceived and developed the SIAPAD system following a decentralized architecture based on the concept of a Spatial Data Infrastructure (SDI) (Coleman and McLaughlin, 1998).

SIAPAD can be considered a pioneer information system in this field. Since the implementation of this type of system requires a significant cooperative effort among different types of organizations (37 technical organizations in the case of SIAPAD), it is difficult to find similar complete and sustainable full implementations of a distributed system for disaster prevention and relief in a multinational region of developing countries. The aim of this paper is to describe the technical decisions that we made and the methods that we designed to develop SIAPAD, together with general conclusions and future directions which we learned as a result of this work. By doing so, we aim to promote the application of this type of technical solution, which could contribute to the final goal of decreasing the risk of disasters, especially in developing countries, with consequent important social and economic positive impacts.

In this paper, we first describe our general design of SIAPAD as a decentralized network following the concept of a SDI. Then, we present the GEORiesgo web-based application, the component of SIAPAD that facilitates information access with an innovative knowledge-based thematic search engine. After this, we describe details about the implementation of the Service-Oriented Architecture and the participating organizations. Finally, we discuss related work, the main achievements, and future lines of work. 


\section{The SIAPAD information system}

As recognized worldwide, sharing spatial data can significantly facilitate the task of disaster management, since most information about disasters has a spatial nature (Amdahl, 2002; Bruzewicz, 2003; Donohue, 2002). Geographic information systems and the internet are useful mechanisms that can facilitate information exchange about disasters (Peng and Tsou, 2003; Crossland et al., 1995; Mansourian et al., 2006). However, usually there are difficulties with the collection, access, dissemination, and usage of required spatial data for disaster management (SNDR, 2002). In the particular case of the Andean Community, we especially confirmed these types of problems after analyzing existing information systems related to disaster risk management, at the beginning of the PREDECAN project (Molina, 2006). Our analysis showed that national governmental organizations and other organizations in the Andean Community individually collect relevant information useful for disaster risk management. However, it was usually difficult to access these sources, or even to know of their existence. In addition, many institutions lacked the policy, equipment, software, and human resources needed to implement appropriate information systems.

As an answer to this problem, we defined the following main requirements for the development of SIAPAD: (1) decentralization, to offer appropriate autonomy and independence to information producers (e.g., the different institutions managing civil defense, meteorology, seismology, demography, etc.), (2) accessibility, to facilitate access to the available information about disaster risk management by different types of users (e.g., analysts, researchers, educators, and general public), and (3) sustainability, to implement a sustainable technical solution, with acceptable costs according to the conditions of developing countries of the Andean Community, furthermore flexible to accept changes and to allow the inclusion of new components in the future. According to these requirements, we designed the SIAPAD system making the following technical decisions:

- Decentralization is supported by a distributed architecture with two levels: server nodes and facilitator nodes. Server nodes publish institutions' local information resources (e.g., maps) and metadata to the internet. Facilitator nodes provide tools for information searching and visualization for a variety of users. The decentralized architecture was designed following the concept of a thematic Spatial Data Infrastructure supported by international standards, for example, ISO (ISO, 2006), INSPIRE (European Union, 2007), and ORCHESTRA (Denzer et al., 2005). This design follows the idea of a Service-Oriented Architecture (SOA) based on the distribution of data and metadata across a network of nodes, linked through standardized web services, which promote interoperability. With this architecture, each server node is a provider that creates its own web services and publishes their interfaces and access information. The facilitators (and potentially other web applications) are service clients that locate entries in the servers' metadata catalogs for binding and invoking their web services. In the case of SIAPAD, this SOA approach was extended by adding digital documents and institutional web pages to the metadata catalogs, so that any information related to disaster management could be found and accessed.

- Accessibility was facilitated with the development of a webbased application (called GEORiesgo), with a particular version for each country (Bolivia, Ecuador, Colombia, and Peru). In

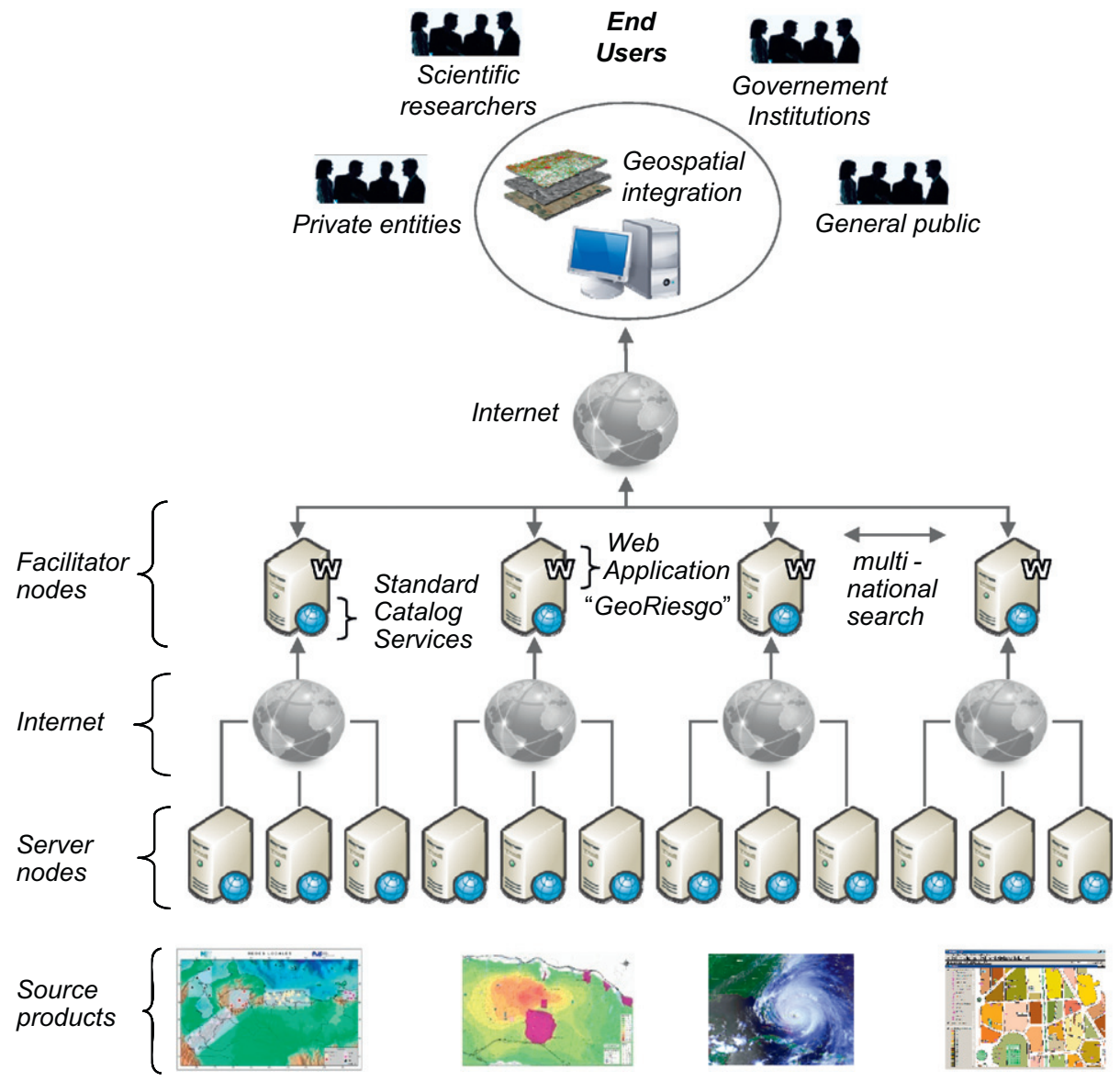

Fig. 1. General architecture of SIAPAD. 
order to provide easy access for different types of users, the web application provides a thematic search engine that helps users who are not familiar with the information sources. This search engine is described in detail in Section 4. Accessibility was also facilitated using a geographic visualization tool that integrates maps based on geographic references, which is an appropriate integration scheme since most information about disasters has a spatial nature.

- Technical sustainability was facilitated by using open source tools (Molina et al., 2008). This is especially important in the Andean Community, a region with limited resources for system development and maintenance. In particular, using open source tools made it easier for information producers to inexpensively publish information. Technical sustainability was also promoted by designing and implementing an open architecture for the thematic search engine. This engine uses a configurable model to support the search (see Section 4). System administrators can modify the content of this model with a convenient software tool, in order to tune or extend the capabilities of the search engine.

Fig. 1 shows the distributed architecture of SIAPAD with the two types of nodes: server nodes and facilitator nodes. Server nodes are set up at the different national institutions that generate useful information for planning and executing tasks oriented to risk management, e.g., civil defense, geophysics, or hydrometeorology. Each local institution is responsible for producing and maintaining the information that it administrates. Furthermore, four national facilitator nodes are set up for Bolivia, Colombia, Ecuador, and Peru. Each facilitator has a national version of the same web application to search and visualize the information. This web portal provides direct access to the information resources and metadata of the corresponding country and also allows for multinational searches by accessing the information collected by other facilitator nodes. Facilitator nodes share a common search model to support the thematic search (see details in Sections 4 and 5) and, as noted above, the model can be modified by administrators to include nation-dependent search options and terminology.

\section{The web application GEORiesgo}

An important component of the SIAPAD architecture is a webbased application for information search and visualization. This web application is called GEORiesgo (riesgo means risk in Spanish) and operates at the facilitator nodes, providing mechanisms for information discovery and visualization. In order to maximize information access, the web application was designed for a wide range of users. This includes both the users who are familiar with disaster risk management tasks and the general public (either from the Andean Community or any other international users). For instance, SIAPAD is directed to decision makers (in local or national governments), territorial planners at the technical level, persons in charge of preparation for and operational attention to disasters, scientific researchers, educators, the media, etc. The web application includes two main components:

- A thematic search engine that guides users through the data search process using a knowledge-based approach (see Section 4 for more details). The search engine translates search goals expressed in language more familiar to general users into search expressions with specific keywords closer to available information sources. Fig. 2 shows the initial window to formulate search goals.

- A geographic viewer that integrates and visualizes geographical information, which is particularly important in the context of disaster risk management. GEORiesgo finds and integrates geographical information from different sources, taking into account that they can contain different geographical representations (e.g., different coordination systems).

Fig. 3 shows how GEORiesgo presents a list of geographic layers corresponding to a particular search and a geographic viewer that integrates and visualizes the selected layers. This integration is useful for analyzing disaster risks, for example, by identifying populated areas near risk zones. GEORiesgo provides this solution using the web geoservice standards Catalog Service for the Web (CSW) and Web Map Service (WMS) generated by the Open Geospatial Consortium (OGC, 2008). The first kind of service (CSW) provides a way to query remote metadata catalogs and, therefore, a way to search repositories with references to data and services, usually about geographical information. The Web Map Service allows remote visualization of published maps by sending images to the client application for the requested spatial extent.

\section{The thematic search engine}

The GEORiesgo application includes a thematic search engine that follows a knowledge-based approach to guide users through the data search process. When users look for information with a search engine, they usually provide a keyword or, in general, a search expression (a set of keywords in a logical expression) and the search engine finds metadata that match the search expressions. This kind of search is useful for finding information especially when the user is familiar with the domain language. However, general users are not always familiar enough with

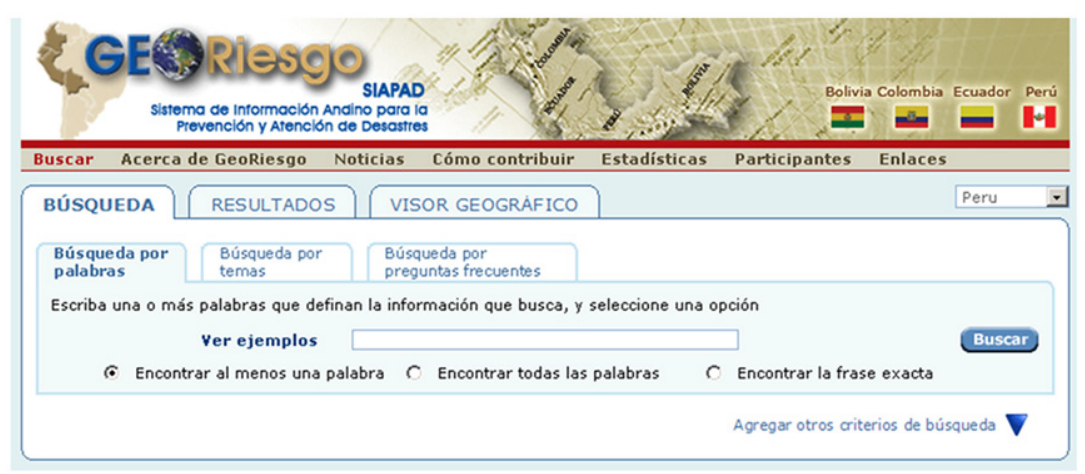

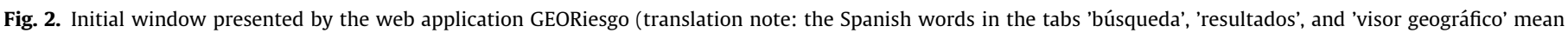
'search', 'results' and 'geographic viewer' respectively). 


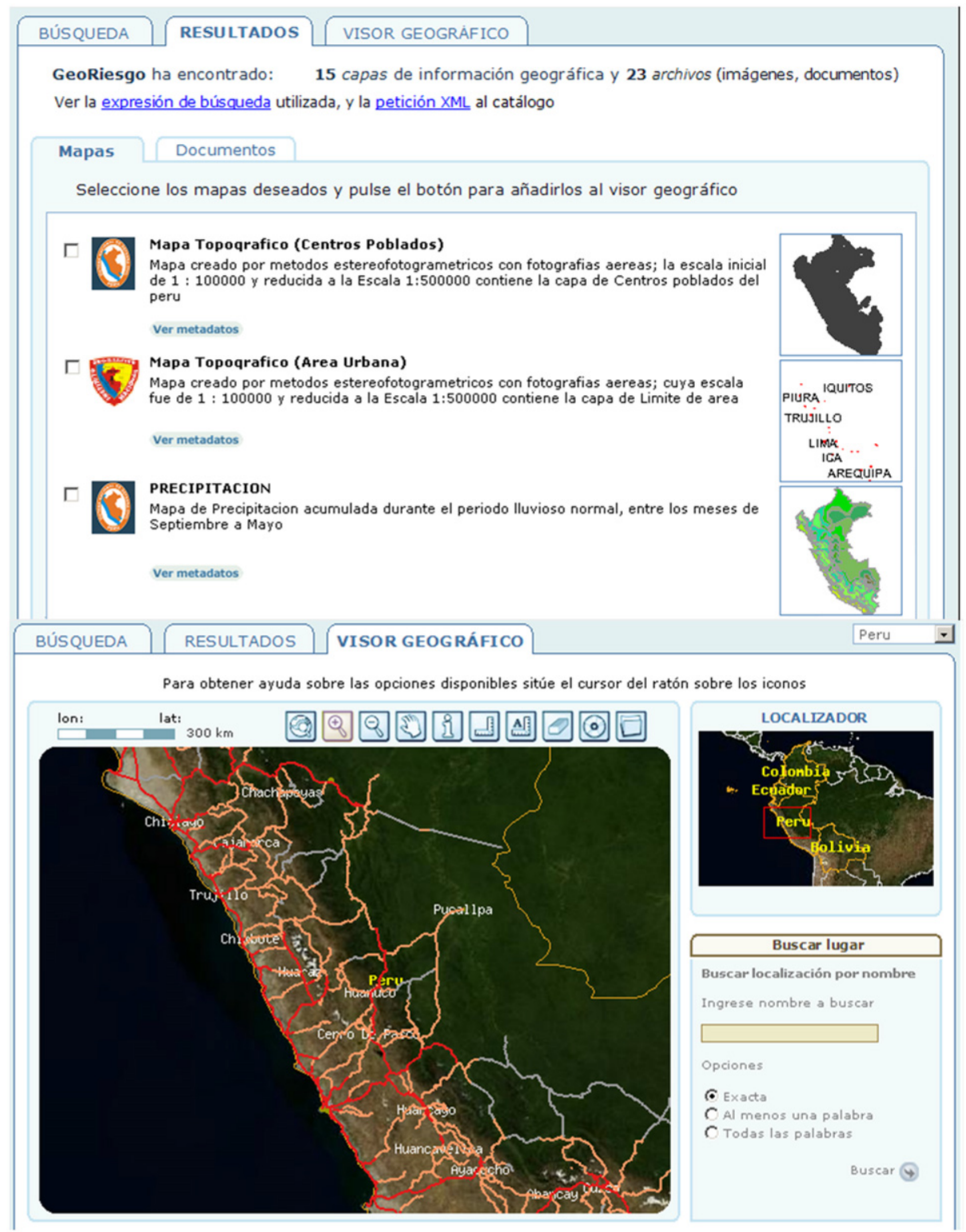

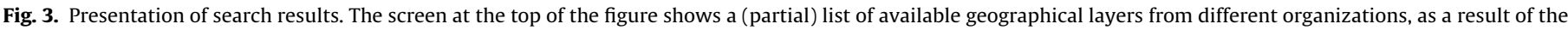
search. The screen at the bottom shows the geographic viewer, which presents an integrated view of the layers selected by the user.

domain languages to write appropriate keywords and search expressions. In the domain of disaster risk management, this happens, for example, because some institutions or software products in the Andean Community are not known by all users. Accordingly, we designed a thematic search engine that automatically generates search expressions with appropriate keywords.

Our method follows a task-oriented approach. This approach assumes that it is easier for users to express what they want to do instead of what they want to search. We distinguish between the user language and the search language. The user language is closer to the information role that defines what the information is needed for, within a particular context that is familiar to the user. On the other hand, the search language includes technical terms and specific names about information sources. For example, the inventory of earthquakes in a particular geographic area could be found with specific keywords such as DesInventar (the name of a web application with this information), which may not be familiar to general users. In this case, users could express more easily what this information is needed for. For example, the inventory of earthquakes could be used to analyze vulnerability of the geographic area (in the context of territorial planning) or for scientific geologic research work in the area.

With our search engine, users define their search criteria using special windows (Fig. 4). In these windows, users formulate search goals related to the information purpose (e.g., a task to be done, a user role, a category of risk management process, etc.) as well as spatial and temporal constraints. Our method translates the search criteria into specific search expressions formulated as a set of keywords in a search expression (Fig. 5). This figure shows an example of a search expression where the keywords are proper names of web applications (e.g., DesInventar) and organizations in the area (e.g., Ingemmet and IOP).

Table 1 shows logic predicates that correspond to the formalization of the search criteria in the user language. Some predicates were designed for specific types of users. For example, the predicate $U \operatorname{serTask}(x, y, z)$, which represents a typical task, can be useful for users who are not familiar with the vocabulary of disaster risk management.

Fig. 6 illustrates how our method generates search expressions. The method includes three main inference steps: (1) check, 


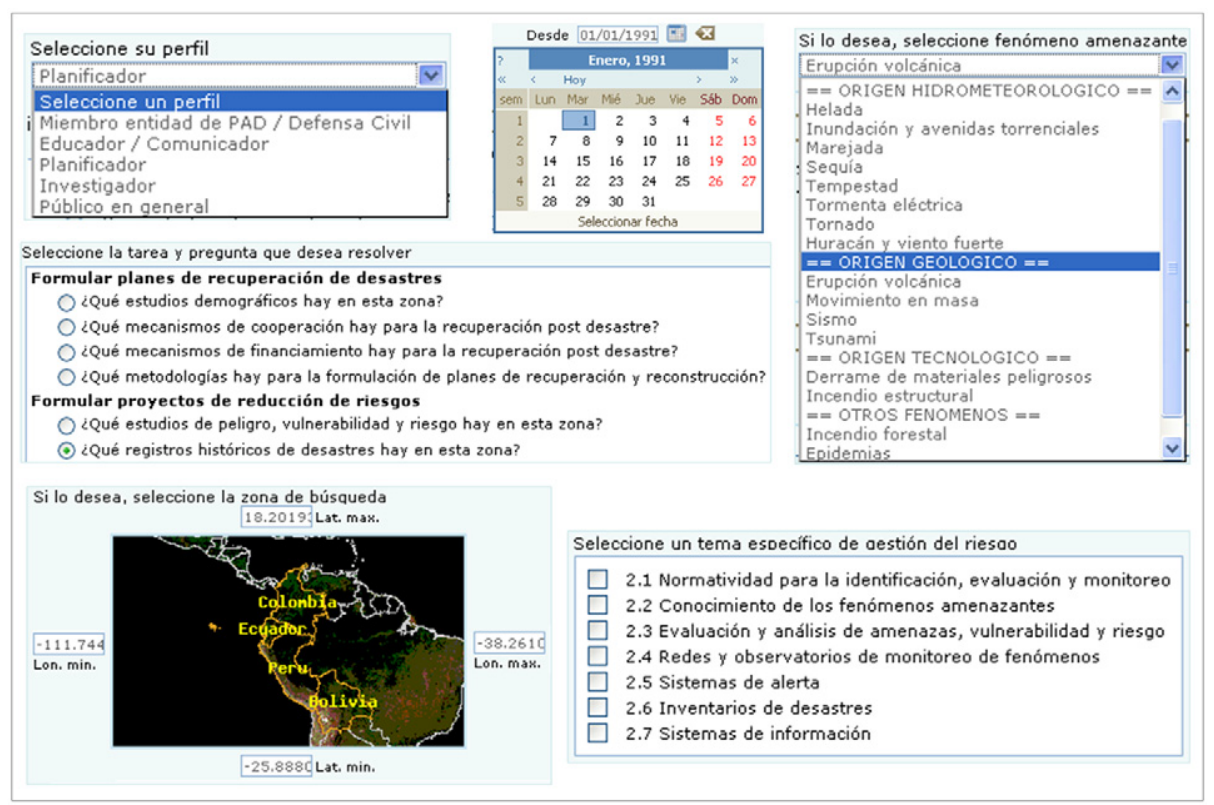

Fig. 4. Examples of windows provided by the web application GEORiesgo to acquire search criteria from the user.

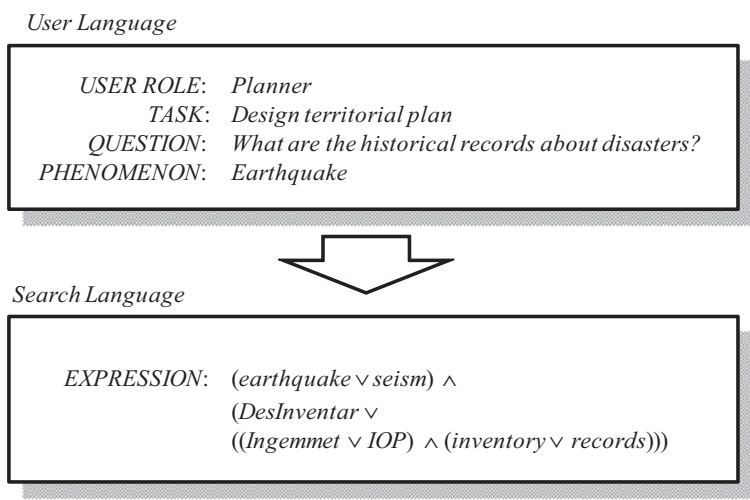

Fig. 5. Example of translation from the user language to the search language.

to check valid search queries and, if necessary, ask the user for additional information, (2) translate, to generate initial search expressions, and (3) expand, to expand search expressions with synonyms and more specific terms, as it is done, for example, by Buttcher et al. (2004). In this example, the user initiates the search with two search criteria (a user task and a phenomenon) and, then, when the system asks for additional information, the user specifies a particular geographical area.

The method uses a heuristic knowledge model that represents human expertise for data searching about disasters and risk management. The formalization of the knowledge model is based on many-sorted first-order logic (Meinke and Tucker, 1993). The model is distributed in three knowledge bases (Fig. 6):

- Combination rules. This knowledge base includes rules for search criteria combinations. This is represented with the general format $\left[c_{i} \wedge\right.$ Conditions $\rightarrow$ Requires $\left.\left(c_{j}\right)\right]$, which means that a particular search criterion $c_{i}$ requires an additional criterion $c_{j}$ to be completely specified. For example, certain questions related to locations require that the user must also provide the spatial area.

- Translation rules. This knowledge base includes rules relating search criteria and search expressions. The general format is $\left[c_{1} \wedge \ldots \wedge c_{n} \wedge\right.$ Conditions $\rightarrow$ KeywordExpression $\left.(e)\right]$, which defines a search expression $e$ (for example, $e=\left(k_{1} \vee k_{3}\right) \wedge$
Table 1

Examples of predicates to formulate search expressions.

\begin{tabular}{|c|c|}
\hline Search criteria & Description \\
\hline $\operatorname{UserTask}(x, y, z)$ & $\begin{array}{l}\text { Task to be done by the user, where } x \text { is the role of } \\
\text { the user (planner, scientific, etc.), } y \text { is a typical task } \\
\text { performed by this type of user, and } z \text { is a typical } \\
\text { question that the user needs to answer to perform } \\
\text { the task. }\end{array}$ \\
\hline RiskManagement $(x, y)$ & $\begin{array}{l}\text { Disaster risk management process, corresponding } \\
\text { to typical categories used in theories of this domain. } \\
\text { Here, } x \text { is a general theme related to disaster risk } \\
\text { management, and } y \text { is a risk management process. }\end{array}$ \\
\hline Phenomenon $(x)$ & $\begin{array}{l}\text { Category of physical phenomenon (earthquake, } \\
\text { flood, etc.) in which the information is classified. } \\
\text { Here, } x \text { is the name of the category. }\end{array}$ \\
\hline SpatialFilter $(x)$ & $\begin{array}{l}\text { Geographical area, where } x \text { is an array with four } \\
\text { values: minimum latitude, maximum latitude, } \\
\text { minimum longitude, and maximum longitude. }\end{array}$ \\
\hline TimeFilter $(x)$ & $\begin{array}{l}\text { A time interval defined with an initial date and a } \\
\text { final date. }\end{array}$ \\
\hline Toponym $(x)$ & $\begin{array}{l}\text { Geographic place, where } x \text { is the name of the place } \\
\text { considering different scales (e.g., city, region, } \\
\text { country, etc.). }\end{array}$ \\
\hline
\end{tabular}

$\left.\left(\neg k_{2} \vee k_{5}\right)\right)$ for a particular combination of search criteria $c_{1}, \ldots c_{n}$.

- Disaster ontology. This knowledge base includes general concepts and relations (e.g., is-a relations and synonyms) about disaster risk management. This ontology is partly based on the Controlled Vocabulary of Disaster Terminology created by the Regional Disaster Information Center-Latin America and the Caribbean (CRID, 2001). At the implementation level, this ontology is represented by using the Resource Description Framework (RDF) format, a World Wide Web Consortium (W3C) specification.

The development of the knowledge model required a significant effort, including the participation of experts in disaster management. We designed special evaluation tests to build and refine successive versions of the model. The tests evaluated search quality according to two parameters: precision $(P)$ and recall $(R)$. These parameters have been used regularly to measure 
1. Initial search criteria (provided by the user)

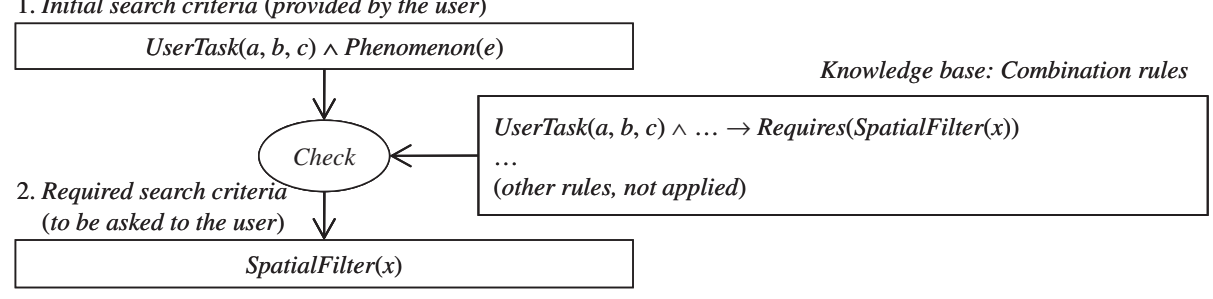

3. Extended search criteria (provided by the user)

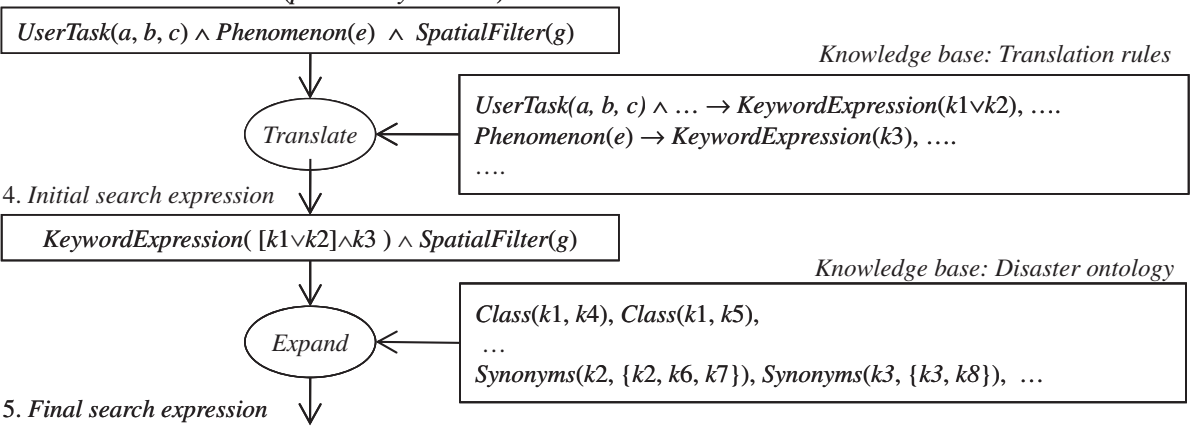

KeywordExpression $([[k 1 \vee k 4 \vee k 5] \vee[k 2 \vee k 6 \vee k 7]] \wedge[k 3 \vee k 8]) \wedge \operatorname{SpatialFilter}(g)$

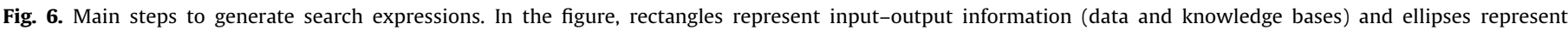
inference steps.

Table 2

Number of keywords and expressions of the search model.

\begin{tabular}{ll}
\hline Elements & Number \\
\hline Main keywords for phenomena & 17 keywords \\
Synonyms for phenomena & 78 keywords \\
Main keywords for disaster risk management & 93 keywords \\
Synonyms for disaster risk management & 244 keywords \\
Search expressions for risk management processes & 36 expressions \\
Search expressions for user tasks & 68 expressions \\
Search expressions for phenomena & 17 expressions \\
Total keywords & 432 keywords \\
Total expressions & 122 expressions \\
\hline
\end{tabular}

the performance of information retrieval and information extraction systems (van Rijsbergen, 1979). Our evaluation method uses the set of prefixed search options $S=\left\{s_{i}\right\}, 1 \leq i \leq k$, provided by the knowledge model (in the final version of the model $k=121$ ).

To calculate $P$, the value $P_{i}=A_{i} / B_{i}$ is obtained for each search option $s_{i}$, where $A_{i}$ is the number of relevant products retrieved by $s_{i}$ and $B_{i}$ is the total number of products retrieved by $s_{i} . P$ is the average of the values $P_{i}$. To estimate the parameter $R$, a set $P=\left\{p_{j}\right\}$, $1 \leq j \leq n$, is obtained as a representative random sample of information products $(n \geq 100)$. The value $R_{i}=C_{i} / D_{i}$ is obtained for each search option $s_{i}$, where $C_{i}$ is the number of products in $P$ that are relevant and retrieved by $s_{i}$ and $D_{i}$ is the total number of products in $P$ that should have been retrieved by $s_{i}$. The value of $R$ is the average of the values $R_{i}$. The values for these metrics range from 0.0 (worst value) to 1.0 (best value). A perfect precision score of 1.0 means that every result retrieved by a search was relevant and a perfect recall score of 1.0 means that all relevant products were retrieved by the search. The final evaluation generated the values $P=0.75$ and $R=0.71$, which are good results compared to the usual values obtained for these metrics in other in-domain retrieval tasks (Lupu et al., 2009).

The four national facilitator nodes share the described search model. The model representation accounts for nation-dependent terminology, such as differences in proper names or certain nouns (for example, the term huaico is used in Perú for avalanche). Users from one country can use their own national terms to find relevant information in other countries (where different terms were used to categorize the information). Table 2 shows the number of keywords and search expressions of the initial generic version of the model. To facilitate the sustainability of the system, the system administrator of each node can modify the model with the help of a web tool. The tool provides a user-friendly user interface that helps administrators to make changes and keep the consistency of the complete model.

\section{The implementation of the Service-Oriented Architecture}

We designed SIAPAD as a Service-Oriented Architecture following the principles of a Spatial Data Infrastructure, with data and metadata distributed across a network of nodes (facilitator nodes and server nodes), and linked through standardized web services which guarantee interoperability (Fig. 7). SIAPAD uses two basic types of services: discovery services and visualization services. For these services, we followed the standard specifications established by the Open Geospatial Consortium (OGC, 2008): Catalog Service for the Web (CSW) and Web Map Service (WMS).

The facilitator nodes, which are installed at the institutional members of CAPRADE in each country (one per country), host customized versions of the GEORiesgo application. Each facilitator also hosts a national catalog with metadata records that are harvested by an automatic process from the institutional catalogs at the server nodes. For this purpose, the facilitator nodes host a catalog server, with the role of managing the catalog (harvesting, permissions, editing) and publishing the CSW search service. With this approach, the national catalogs are accessible via CSW not only by the GEORiesgo application but also by other SDI clients.

The search engine of the GEORiesgo application uses the CSW service at each national facilitator node to search for metadata records in the national catalog hosted in the same node. This allows the application to quickly perform searches at the national 


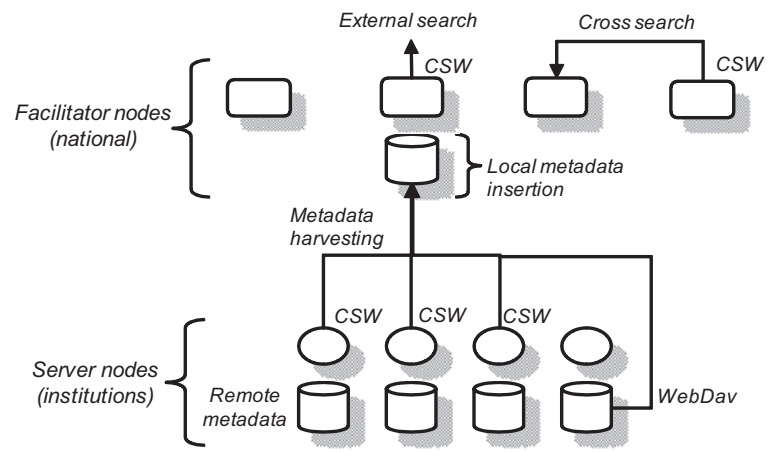

Fig. 7. Metadata catalogs and CSW search services in the SIAPAD architecture.

level. The search engine selects metadata records that match input search expressions, using selected fields (e.g., title, abstract, keywords, in addition to spatial and temporal constraints). The search engine can also connect to the other facilitator nodes for multinational searches, as well as to any other external catalog configured by the node administrator.

Implementation of the software components at the facilitator nodes was guided by the goal of using open source tools, already very mature and extended in the geospatial realm. The programming language was Java and the main open source software packages used were Geonetwork (metadata manager and CSW server), MapBuilder library (map viewer), PostgreSQL/PostGIS (spatial database management), Apache (web server), and ExtJS library (graphical widgets).

The key to the project's success resided in server nodes providing WMS and CSW services. A total number of 26 server nodes to publish information were installed; some of them were shared by different organizations. Since most of the organizations had never previously published these web services before the PREDECAN project, they followed a training program for data publishing together with continuous technical support provided during the project. In addition to installation and use of software tools, the training program included a set of practical recommendations (Vargas et al., 2008a) about the use of standards for metadata and service publishing. For example, we followed the standards ISO 19115 (about information fields) and ISO 19139 (about exchange formats) to be used by metadata records. In addition, some practical recommendations dealt with organizing layers in WMSs, publishing map legends and feature information, and using a standard vocabulary in the metadata keywords (this vocabulary was implemented in the project in RDF format to be used by the Geonetwork metadata manager).

The same open source technologies used in the facilitator nodes (e.g., PostgreSQL, Geonetwork, MapServer and gvSIG) were recommended for use in the server nodes, providing a fully free and open solution. Some institutions also successfully integrated existing proprietary software systems into the SIAPAD network. For some institutions, the deployment of a metadata database and CSW server was not deemed to be worth the effort (set up and maintain additional software to publish a few metadata records) so in those cases an alternative mechanism was suggested via WebDAV folders, which are just file folders in the server nodes with metadata records stored in XML files. The Geonetwork software at the national facilitator nodes was able to harvest these folders and integrate their metadata into the national catalog.

Technical tests were carried out to guarantee the correct operation of the whole system and to validate operational performance. This included a test with stress simulation software to evaluate the system performance with simultaneous requests. This evaluation showed acceptable answer times according to the technical requirements of the system, which was designed for a maximum of 1000 concurrent users. For instance, with 100 simultaneous users, the average answer time was $0.51 \mathrm{~s}$, increasing to $2.8 \mathrm{~s}$ (still, within acceptable limits) for 1000 concurrent requests. These results were obtained when the system was installed at the end of 2008. The tests were performed using a slow-average Internet connection as available in the region. The CSW protocol was also a time cost factor (regarding classification of results, obtaining thumbnail previews of map services, etc.). This had to be solved on the client side and therefore caused delay in presenting search results. However, this was considered preferable to the use of a nonstandard catalog search service.

\section{Participating organizations}

The development of the SIAPAD system was initiated in November 2005 and was completed at end of the PREDECAN project in June 2009. A first version of a fully operational version of the system was installed at the end of 2008. During the development of SIAPAD it was essential to have the active participation of organizations corresponding to information providers and potential user (Table 3 ).

These organizations participated in the following ways:

- Collaborative design. Frequent international workshops about SIAPAD were organized with all the participants to collect opinions and requirements and to validate the partial and final technical solutions (e.g., about the user interface and the search language). The workshops were periodically organized and coordinated by the management team of the PREDECAN project.

- Information publishing. The organizations followed a training program for information publishing with continuous technical support. Most of the organizations had never previously published WMS or CSW services. As a result of the development of SIAPAD, these organizations are part of the network of 5131 information products that are now accessible through the GEORiesgo web application (see Table 4).

Table 3

Participating organizations in the SIAPAD network publishing disaster-related information.

\begin{tabular}{lll}
\hline Countries & Categories & Organizations \\
\hline \multirow{2}{*}{ Bolivia } & Civil defense and risk management & VIDECICODI \\
& Geology and geophysics & SERGEOTECMIN, OSC \\
& Hydro-meteorology & SENAMHI \\
& Statistics and planning & INE, MPD \\
& Geographic institutes & IGM \\
& Others & SIA, GMLP, SNA \\
Colombia & Civil defense and risk management & DPAD \\
& Geology and geophysics & INGEOMINAS \\
& Hydro-meteorology & IDEAM \\
& Statistics and planning & DANE, DPN \\
& Geographic institutes & IGAC \\
& Others & DPAE, DCC, HUMBOLDT \\
& Civil defense and risk management & STGR \\
& Geology and geophysics & SGN, IGEPN \\
& Hydro-meteorology & INAMHI \\
& Statistics and planning & INEC, SENPLADES \\
& Geographic institutes & IGM \\
& Others & CLIRSEN, MAE, DMSC \\
& Civil defense and risk management & INDECI \\
& Geology and geophysics & INGEMMET, IGP \\
& Hydro-meteorology & SENAMHI \\
& Statistics and planning & INEI \\
& Geographic institutes & IGN \\
& Others & MTC, PREDES \\
& & \\
& & \\
& & \\
& & \\
& & \\
& &
\end{tabular}


Table 4

Number of information products (published with metadata by information providers) for each risk management category and country.

\begin{tabular}{lcccc}
\hline Risk management categories & Bolivia & Colombia & Ecuador & Peru \\
\hline $\begin{array}{l}\text { Policy instruments } \\
\text { Risk identification, evaluation, and }\end{array}$ & 285 & 260 & 311 & 357 \\
$\quad$ monitoring & 239 & 551 & 484 & 840 \\
$\begin{array}{l}\text { Education and socialization for risk } \\
\quad \text { management }\end{array}$ & 75 & 135 & 124 & 256 \\
$\quad \begin{array}{l}\text { Reduction of risk factors } \\
\begin{array}{l}\text { Preparation for emergency response and } \\
\quad \text { relief }\end{array}\end{array}$ & 60 & 68 & 124 & 213 \\
$\quad \begin{array}{l}\text { Recovery, rehabilitation, and } \\
\quad \text { reconstruction }\end{array}$ & 82 & 27 & 71 & 89 \\
\hline
\end{tabular}

SIAPAD also integrated other multinational information systems in the Andean region, which provide more specialized information (Vargas et al., 2008b). For example, (1) DesInventar is a distributed system with historical inventories of disaster effects (Velásquez, 2005); (2) Geosemantica (Escallón, 2005) is a web-based collaborative workspace for spatial information (e.g., satellite images, geological information, infrastructures, and power stations), and (3) the BiVa-PaD network (disaster risk and prevention relief digital libraries) (López, 2007) is a digital library with documents about disasters in Latin America and the Caribbean Region. SIAPAD integrated this information by using open standards for interoperability. For example, conversion tools were developed to transfer BiVa-PaD library records to ISO 19139 and DesInventar data were published as maps with the WMS standard and properly cataloged.

\section{Related work}

Spatial Data Infrastructures have evolved in recent years from a theoretical framework to a legal and operational reality at many levels of organization. At the local level, municipalities all around the world, from Palestine (El-Atrash et al., 2008) to Perú (Murgia et al., 2002), are now using GIS connected to geoservices to manage local resources and provide public services. In the European Union, the United States and other regions, national and multinational legal frameworks like the European Union INSPIRE Directive have also trickled down to local entities (Birch, 2010).

At the national level most developed countries now have their national data infrastructure in place, typically by federating regional/local SDIs and adding geoservices provided by the national government institutions. Prototypical examples are the US geodata.gov and the national SDI in Spain spanned from INSPIRE (Mezcua-Rodríguez, 2009), sometimes with a strong involvement of the private sector. Most interesting, thematic SDIs, some of them focused on natural resources management, have been created on the base provided by the national SDI (Guimet, 2004; Strande, 2009).

In addition to the INSPIRE framework, there are other initiatives at the multinational level like the UNSDI (Henricksen, 2007) are tackling the construction of multinational SDIs, but the results are so far quite limited. Since natural resources, weather, or disasters do not know of political boundaries, thematic SDIs are a natural scenario for the building of transnational infrastructures, but the ongoing efforts face bureaucratic and administrative barriers. In this context, SIAPAD is one of the very few examples of a fully implemented thematic SDI at the multinational level in developing countries. It is important to note that SIAPAD has not been built on the base provided by national SDIs but, on the contrary, has itself become the foundation for those national SDIs. The development of SIAPAD is an example of the potential of SDIs for multinational cooperation which at the same time is useful at the national level.

SIAPAD provides the thematic web application GEORiesgo for information search and visualization in the domain of disasters. Other web-based applications for search and visualization have been developed in this context in developing countries. For example, the SERVIR system (Cherrington, 2007) is a groundbreaking initiative, which provides access to a wealth of data resources in Central America and Africa. In comparison with GEORiesgo, the SERVIR system is not fully developed under the service-oriented framework of SDIs. In the context of SDI-based systems, Mansourian et al. (2006) developed a prototype webbased system on SDI as a pilot project in Iran to facilitate disaster risk management. In contrast to this system, GEORiesgo is not a prototype but a fully operational implementation and it follows a multinational approach. Totolhua et al. (2008) developed the PDGP web application on SDI in Mexico to facilitate disaster prevention. In contrast to PDGP, GEORiesgo includes other risk management processes and a multinational approach. In addition, compared to these web-based applications, an original contribution of GEORiesgo is its search model that helps users to formulate search queries. The model includes combination/translation rules and a disaster ontology (see Section 4). GEORiesgo uses the model to operate as an expert search tool specialized in disasters that provides easier access for different types of users, taking into account differences in nation-dependent terminology.

\section{Discussion}

The development of SIAPAD contributed to the development of a complete thematic SDI in the Andean Community in the following way:

- Culturing information sharing. As a result of the development of SIAPAD, a total of 37 organizations participate as information providers. They provide public access through web services and standard metadata to their information products. This was facilitated by the development of a multinational training program for these organizations with detailed recommendations on web services configuration and metadata creation, and continuous technical support for the organizations. Before the development of SIAPAD, there were not efficient mechanisms for information sharing other than coordination committees, some individual agreements, and web pages with limited information. For certain institutions, selling data is part of their business model, so they are not able to publish free datasets. However the use map services, which do not transfer data but rather map images, is well accepted by these organizations. Although this may seem obvious in other countries, the public access to maps is an important step toward the integration and exchange of geoinformation in the Andean region.

- Creating an access network. SIAPAD was designed as a ServiceOriented Architecture with 26 server nodes and 4 facilitator nodes. The facilitator nodes include the web-based application GEORiesgo for information search and visualization. The GEORiesgo web application includes an innovative search engine with a knowledge-based solution that assists users to find information sources and a geographic viewer to visualize together maps from different sources and complementary thematic content in an integrated view.

- Standardization. The development of SIAPAD promoted the use of standard web services (e.g., WMS and CSW). An ontology of 
disaster-related terms was also created (in RDF format for the Geonetwork metadata manager) to help systems administrators to select keywords for metadata. The development of SIAPAD also contributed to identify differences among data representation (such as scale and symbols), especially in different countries. This is useful to establish data harmonization strategies.

Concerning the impact of this work in the disaster risk management processes, SIAPAD helps to make decisions related to disasters, providing accessibility to valuable information from multiple sources. The geographic viewer that integrates different geographic layers (hazards, population, infrastructures, etc.) makes it easier for end users to analyze diverse information about disaster risks. The integration of information from neighboring countries enables users to make decisions related to phenomena spreading across the borders (e.g., seismic or hydrometeorological phenomena). In addition to that, SIAPAD is also useful for observing the degree of implementation of risk management processes in each country. SIAPAD shows the available information corresponding to the different categories of risk management processes and, also, given the uniform multinational approach, permits users to compare the availability of this information in each country. This contributes to increasing awareness in the region about the benefits of disaster risk management processes, promoting the implementation of such processes without duplicated efforts.

Regarding future work related to SIAPAD, additional activities could be done in the following areas:

- Data harmonization. SIAPAD helped to identify differences in methodology, content, and categorization among disasterrelated datasets. For instance, different countries use varied methods to calculate and represent derived data, like seismic risk, and in addition they are classified and displayed using different rules. Thus, when the corresponding map services are seen together, they do not match at the borders and can create confusion for the users. Therefore, additional harmonization tasks should be carried out to facilitate the integration of these information sources.

- Web services. Besides the WMS and CSW standard web services promoted by SIAPAD, other standard geoservices (Web Feature Service (WFS) for raw vector data access, Web Coverage Service (WCS) for raw raster data access and Web Processing Services (WPS) for server-side analysis) could be implemented in the future for geoprocessing-based analysis applications like risk assessment and real-time emergency impact evaluation. In addition, initiatives based on on-the-ground sensor networks (seismic, volcanic, hydrometric, etc.) should be explored to provide additional services based on open standards like Sensor Web Enablement (SWE) and Common Alerting Protocol (CAP). Furthermore, it is expected that mature technical solutions from the field of the Semantic Web (Berners-Lee et al., 2001) in the future could be used to promote information sharing with the help of common ontologies.

- Shared infrastructures. Especially in developing countries, using shared infrastructures provided by institutions is a useful strategy for organizations that are not able to afford the cost of implementing and maintaining their own information services. This goal can be achieved with the help of new tools to publish geoinformation in a cloud-based infrastructure instead of using hosted nodes, as promoted by Geosemantica (Escallón, 2005).

- Information management. The development of SIAPAD helped to improve the organization of information within the different participating institutions, making the available datasets more accessible within these institutions. However, especially in developing countries, some institutional processes related to information management are weakly defined, which can significantly affect the sustainability of the system. Thus, these tasks should be formalized in more depth as an essential part of each institution's strategic plans and daily operations.

\section{Conclusions}

In summary, the main goal of SIAPAD was to increase the visibility and accessibility of information to improve disaster risk management in the Andean Community, a region frequently affected by disasters linked to the occurrence of natural phenomena (earthquakes, volcanoes, storms, etc.). We designed SIAPAD as a SDI-based architecture, which includes 26 server nodes and 4 facilitator nodes. We also developed the web-based application GEORiesgo for information search and visualization, using an innovative thematic knowledge-based search engine that helps users to find information sources. The development of SIAPAD contributed to the development of a culture of resource sharing in the region, with a total of 37 organizations from four different countries participating as providers of information products about disasters, made available through standard web services (e.g., WMS, CSW) and standard metadata.

The PREDECAN project finished in June 2009 with a complete implementation of SIAPAD. SIAPAD can be considered a pioneer work in the sense that it is a fully operational system to share information about disasters across the Andean Community, a multinational developing region. It can be used as a model for the future development of information systems for disaster management and other related domains for other geographical areas (for example, in Central America, an information system is being built based on the SIAPAD model). An extensive application of this technology can contribute to improve the disaster risk management, especially in developing countries, with consequent important social and economic positive impacts.

\section{Acknowledgments}

The development of SIAPAD was made possible thanks to the financial support of the European Commission and the General Secretary of the Andean Community by means of the PREDECAN Project (ASR/B7-3100/99/313). The project management team of PREDECAN (in particular, Ana Campos and Rubén Vargas) promoted and coordinated the participation of the different actors and also supervised and supported with valuable comments the design and implementation of the software of SIAPAD. The company World Development Consultants (WDC) from Spain was in charge of the international technical assistance in the project. An exploratory prototype of SIAPAD oriented to capture functional requirements was developed in the project in 2007 by the company Sun Gemini from Colombia. The authors thank Amanda Stent for her useful comments on earlier versions of this paper.

\section{References}

Amdahl, G., 2002. Disaster Response: GIS for Public Safety. ESRI. Redlands. CA., http://www.esri.com/news/arcnews/winter0102articles/gis-homeland.html (accessed April 21, 2010).

Berners-Lee, T., Hendler, J., Lassila, O., 2001. The Semantic Web. Scientific American Magazine. May.

Birch, J., 2010. NeoSDI for local governments. In: Proceedings of the GeoWeb Conference. Vancouver, Canada. 
Bruzewicz, J., 2003. Remote sensing imagery for emergency management. In: Cutter, S.L., Richardson, D.B., Wilbanks, T.J. (Eds.), Geographical Dimension of Terrorism. Toutledge, New York.

Buttcher, S., Clarke, C., Cormack, G., 2004. Domain-specific synonym expansion and validation for biomedical information retrieval (MultiText experiments for TREC 2004). In: Proceedings of the 13th Text Retrieval Conference. Gaithersburg, Maryland.

Cherrington, E.A., 2007. SERVIR: An Integrated Platform for the Visualization and Monitoring of the Air, Land and Sea of Mesoamerica. http://www.servir.net (accessed October 15, 2010).

Coleman, D.J., McLaughlin, J., 1998. Defining global geospatial data infrastructure (GGDI): components, stakeholders and interfaces. Geomatica, Canadian Institute of Geomatics 52 (2), 129-144.

CRID (Regional Disaster Information Center-Latin America and the Caribbean), 2001. Disasters Controlled Vocabulary. http://www.crid.or.cr/crid/ing/vocabu lario_controlado_ing.shtml. (accessed April 21, 2010).

Crossland, M.D., Wynne, B.E., Perkins, W.C., 1995. Spatial decision support systems: an overview of technology and a test of efficacy. Decision Support Systems 14, 219-235.

Denzer, R., Güttler, R., Schimak, G., Usländer, T., Atkinson, M., 2005. ORCHESTRA: development of an open architecture for risk management in Europe. In: Proceedings International Symposium on Environmental Software Systems ISESS 2005. Sesimbra, Portugal.

Donohue, K., 2002. Using GIS for All-Hazard Emergency Management. Department of Natural Resources Science, University of Rhode Island, Fall 2002.

El-Atrash, A., Al-Halaybeh, H., Zboun, I., 2008. Spatial data infrastructure towards E-municipality - the case of Beit Sahour municipality. In: Proceedings of the First International Conference on Urban Planning in Palestine. An-Najah National University.

Escallón, J., 2005. GeoSemantica as a technological platform to develop the Colombian spatial data infrastructure. In: Proceedings of GSDI-8. El Cairo Egypt.

European Union, 2007. Directive 2007/2/EC of the European Parliament and of the Council of the European Union of 14 March 2007 establishing an infrastructure for spatial information in the European Community (INSPIRE). Official Journal of the European Union, 25 April 2007.

Guimet, J., 2004. Thematic SDIs: a way to spread out the benefits of interoperability and to enhance the development of regional SDIs. In: Proceedings of Tenth EC GI and GIS Workshop. Warsaw, Poland, 23-25 June 2004.

Henricksen, B., 2007. UNSDI compendium: a UNSDI vision, implementation strategy and reference architecture. In: Proceedings of United Nations Geographical Information Working Group (UNGIWG). http://www.ungiwg.org/ unsdi.htm. (accessed October 15, 2010).

ISDR (International Strategy for Disaster Reduction, United Nations), 2005. Hyogo framework for action 2005-2015: building the resilience of nations and communities to disasters. In: Proceedings of World Conference on Disaster Reduction. Kobe, Hyogo, Japan18-22 January 2005.

ISO (International Organization for Standarization), 2006. Standard ISO/TC 211.Geographic Information/Geomatics. http://www.isotc211.org. (accessed April 21, 2010)

López, I., 2007. BiVa-PaD: An Andean Initiative to Manage Disaster Prevention and Response Information. EIRD Magazine, November 14, 2007.
Lupu, M., Piroi, F., Huang, X., Zhu, J., Tait, J., 2009. Overview of the TREC 2009 chemical IR track. In: Proceedings of TREC (Eighteenth Text Retrieval Conference) 2009.

Mansourian, A., Rajabifard, A., Valadan Zoej, M.J., Williamson, I., 2006. Using SDI and web-based system to facilitate disaster management. Computers and Geosciences 32 (3), 303-315.

Meinke, K., Tucker, J.V., 1993. Many-Sorted Logic and Its Applications. John Wiley and Sons, Inc., Chichester, England.

Mezcua-Rodríguez, J., 2009. The spatial data infrastructure of Spain as an example of success in Europe. In: Proceedings of Ninth United Nations Regional Cartographic Conference for the Americas. New York, 10-14 August 2009.

Molina, M., 2006. Análisis de Sistemas de Información de Prevención y Atención de Desastres en la Comunidad Andina. PREDECAN Project (Apoyo a la Prevención de Desastres en la Comunidad Andina). Technical Report. http://www.comu nidadandina.org/predecan/doc/centro/anal_sist_can.pdf. (accessed April 21 2010).

Molina, M., Bayarri, S., Vargas, R., 2008. The Andean information system for disaster prevention and relief: a case study of multi-national open-source SDI In: Proceedings of FOSS4G (Free and Open Source Software for Geospatial) Conference. Cape Town, South Africa.

Murgia, J., Amemiya, N., Turkstra, J., 2002. Local spatial data infrastructure, Trujillo-Peru. Proceedings of GISDECO Seventh International Seminar on GIS in Developing Countries. ITC, Enschede, The Netherlands, 15-18 May 2002.

OGC (Open Geospatial Consortium Inc.), 2008. OGC Reference Model. http://portal. opengeospatial.org/files/?artifact_id=31112. (accessed April 21, 2010).

Peng, Z.R., Tsou, M.H., 2003. Internet GIS. John Wiley and Sons Inc., NJ.

SNDR (Subcommittee on Natural Disaster Reduction), 2002 A national hazards information strategy: reducing disaster losses through better information. In: Proceedings of National Science and Technology Council, Committee on the Environment and Natural Resources. Washington, DC, April 2002.

Strande, K., 2009. Spatial data infrastructure as tools in environment and geohazard management. Examples from Norway. In: Proceedings of Seventh FIG Regional Conference: Spatial Data Serving People: Land Governance and the Environment-Building the Capacity. Hanoi, Vietnam, 19-22 October 2009.

Totolhua, V.R., Zepeda, O., Muñoz M., Torres, L., 2008. Developer of geospatial portal for the Mexico's disaster prevention with data infrastructure approach. In: Proceedings of Tenth International Conference for Spatial Data Infrastructure. GSDI 10. St. Augustine, Trinidad, February 25-29.

van Rijsbergen, C.V., 1979. Information Retrieval second ed. Butterworth, London.

Vargas, R., Weykam, S., Bayarri, S., 2008a. Recomendaciones sobre difusión de datos para apoyo a la gestión del riesgo. Technical Report-PREDECAN Project (Apoyo a la Prevención de Desastres en la Comunidad Andina).

Vargas, R., Molina, M., Bayarri, S., Campos, A., 2008b. The Andean information system for disaster prevention and relief SIAPAD: an initiative for the development of a thematic SDI. In: Proceedings of Global Spatial Data Infrastructure Conference GSDI 10. St. Augustine, Trinidad; February 25-29, 2008.

Velásquez, A., 2005. DesInventar, databases and applications, Latin America and Caribe. In: Proceedings of World Conference on Disaster Reduction. Kobe Hyogo, Japan18-22 January 2005. 